Qingwei Bai $a^{*}$, Ming Wen $b$

a. Key Laboratory of Integrated Exploitation of Bayan Obo Multi-Metallic Resources, Inner Mongolia University of Science and Technology, Baotou, 014010, P.R. China.

b. Inner Mongolia Autonomous Regional Engineering Technology Research Center of Sustainable Exploitation of Rare Earth Secondary Resources, Inner Mongolia University of Science and Technology, Baotou, 014010, P.R. China.

*Corresponding author: Qingwei Bai Ph.D

Email: abcqingwei@163.com, Tel.: +8615947629690

Address: No.7 Arding Street, Baotou, Inner Mongolia, 014010, P.R. China

Contributions: Experiment and result analysis

Co-author: Ming Wen Ph.D

Email:452779532@qq.com

Address: No.7 Arding Street, Baotou, Inner Mongolia, 014010, P.R. China

Contributions : Experiment and result analysis

\title{
Declaration of interests
}

The authors declare that they have no known competing financial interests or personal relationships that could have appeared to influence the work reported in this paper.

Abstract: A comparison of tailing-based nanocrystalline glass-ceramics prepared by microwave and conventional heat treatment has been made to investigate the effect of slightly microstructure differences on the mechanical properties and thermal stability. Contrast conventional heat treatment, the flexural strength of the samples increased from $169 \mathrm{MPa}$ to $219.9 \mathrm{MPa}$ under the microwave radiation, and the temperature of the hemisphere-stage increased by $81{ }_{\circ} \mathrm{C}$ during the melting. The main factors affecting materials properties under microwave radiation are the increased $\mathrm{Si}-\mathrm{O}$ stretching vibrations frequency and bond force, and the substitution effectively of $\mathrm{Ce}_{3+} / \mathrm{Ce}_{4+}$ in the diopside during crystallization. Besides, the polymeric character of silicon-oxygen network can be reduced by 
microwave radiation to promote the nucleation. Microwave radiation is an energy-efficient technology for the preparation of rare earth tailing-based material.

Keywords: microwave radiation; nanocrystalline glass-ceramics; flexural strength; thermal stability; microstructure differences; Nucleation

\section{Introduction}

Bayan Obo mine is a large polymetallic paragenetic deposit with valuable elements such as iron, rare earth, niobium, which is famous for its containing niobium and rare earth resources in the world. Since the 1950s, the East Bayan Obo mine has been mainly used as iron ore, while most of the symbiotic rare earth, niobium and other strategic resources have to be piled into the tailings dam. The preparation of tailing-based nanocrystalline glass-ceramics by microwave heat treatment opens up a new path for exploit of secondary resources and develops high value-added materials in recent years. Basic compounds $\mathrm{SiO}_{2}, \mathrm{Al}_{2} \mathrm{O}_{3}$, Cao, etc. in the Bayan Obo iron tailing and the strengthening element such as rare earth and niobium , can be used as raw materials for special nanocrystalline glass-ceramics which exhibit high mechanical strength, great corrosion resistance and superior thermal shock resistance. Combined with the characteristics of microwave material preparation technology, an emerging industry field is formed, which is based on the tailing comprehensive utilization and supported by clean and efficient microwave technology.

Over the past decade, hundreds of compounds have been synthesized using microwave irradiation. Microwave heating technology is fundamentally different from the traditional heating method in both theory and practice. The reaction process of the system is affected by microwave radiation, resulting in product or structure differences[1]. In most cases, microwave irradiation technology results in a reduction in reaction time and reaction temperature, for example, in the synthesis of $\mathrm{LaCrO}_{3}[2]$, $\mathrm{GaAlO}_{3}[3], \mathrm{Li}_{0.35} \mathrm{La}_{0.55} \mathrm{TiO}_{3}[4]$, etc. The most interesting phenomenon is that microwave heating can improve the properties of the products, which means the novel or metastable structures materials can be obtained by electromagnetic-material coupling; Microwave synthesis can promote the catalytic activity of oxides (such as perovskite), $\mathrm{La} 1-\mathrm{xAxMnO} 3.15(\mathrm{~A}=\mathrm{Sr} / \mathrm{Ag} ; \mathrm{X}=0 / 0.2)$ synthesized by microwave radiation can greatly enhance the catalytic combustion of methane[5]; The size of precipitated particles is reduced under microwave heating in some situation, for example, microwave technology can rapidly synthesize 5-10 nm nano-zirconia particles; Microwave synthesis can also produce larger particle size 
than traditional synthesis to influence the performance of the material, for example, cerium-stabilized yttrium-aluminum-garnet (YAG: Ce) with large grain size which is synthesized by microwave heating can improve its luminous intensity in yellow light-emitting diode[6]; Binner et al.[7] demonstrated that the microstructure of ceramics is very sensitive to microwave radiation. Yttrium-stabilized zirconia (YSZ) synthesized in a microwave furnace shows an intergranular fracture, while synthesized in conventional method, follows a transgranular fracture. In the microwave radiation field, it is a key step to clarify the evolution of phase composition and microstructure to reveal the reaction mechanism. In this study, by controlling the crystal size, the microstructure differences of Baya Obo tailing-based nanocrystalline glass-ceramics was treated under microwave and conventional heating was analyzed, so as to investigate the mechanism on the improvement of mechanical and high-temperature properties by microwave radiation.

\section{Experimental details}

By using Bayan obo iron ore tailing as the main raw material in the experiment[8], the basic chemical components of nanocrystalline glass-ceramics were obtained by adding appropriate chemical reagents such as $\mathrm{SiO}_{2}$ and $\mathrm{CaO}$ to the tailing, as shown in table 1 . The powder raw material was melted at $1450 \mathrm{o}$ C for 3 hours, and then casted into metal mold to prepare the basic glass precursor material. Microwave and conventional heat treatment are carried out for the basic glass respectively, and schematic of the microwave applicator is shown in Fig. 1. By controlling the heat treatment process, nanocrystalline glass-ceramics with similar grain size were obtained to reduce the influence of grain size difference on microstructure analysis. Microwave heat treatment: nucleation at $690 \mathrm{o}$ for $20 \mathrm{~min}$, crystallization at $750{ }_{\circ} \mathrm{C}$ for $20 \mathrm{~min}$; Conventional heat treatment: nucleation at $690 \mathrm{\circ}$ for 2 hours, crystallization at $800 \mathrm{o}$ for 2 hours. The three-point flexural strength of the glass-ceramics was measured by using universal testing machines, and 3 samples $(3 \times 3 \times 4 \mathrm{~mm} 3)$ were tested for each process. The surface morphology was evaluated at room temperature by laser confocal microscope (LSM, LASERTEC VL2000DX), whose the limited resolution is $0.14 \mu \mathrm{m}$. The thermo-softening of the samples was observed by using hot stage microscope (HSM, YONEKURA, SVF17QP), and the samples was heated to $1300^{\circ} \mathrm{C}$ at $5^{\circ} \mathrm{C} / \mathrm{min}$. Structural analysis was done using a X-ray diffractometer (XRD, BRUKER D8 ADVANCE), the scanning voltage and speed are respectively $40 \mathrm{kV}$ and $0.5^{\circ}$ min. The Raman spectra was recorded on a Raman spectrometer (Jobin Yvon, HR 800) in the wave number range of 50-1200 cm-1 using an excitation source of $\mathrm{Ar}+$ laser $(514.5 \mathrm{~nm})$. 


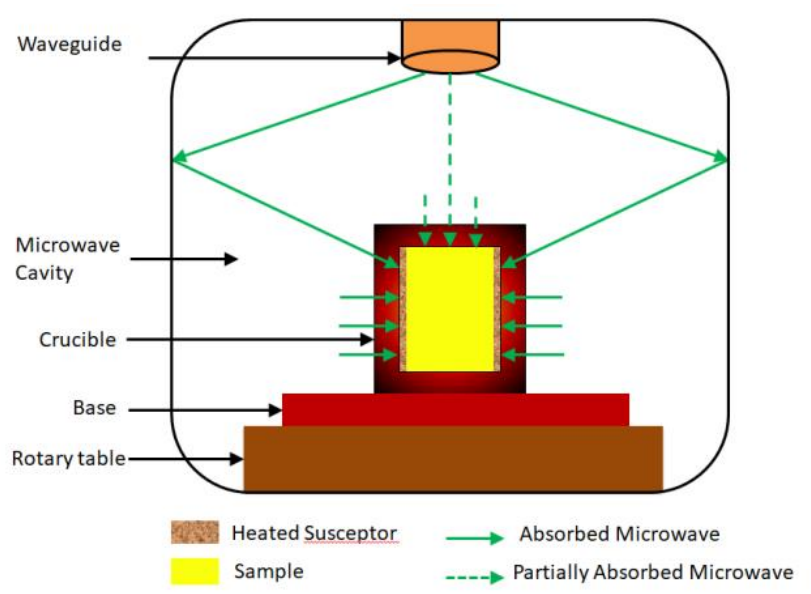

Fig.1 Schematic of the microwave applicator

Table 1 Chemical composition of the glass matrix prepared from the tailing

\begin{tabular}{cccccccccc}
\hline $\mathrm{SiO}_{2}$ & $\mathrm{CaO}$ & $\mathrm{MgO}$ & $\mathrm{Al}_{2} \mathrm{O}_{3}$ & $\mathrm{Fe}_{2} \mathrm{O}_{3}$ & $\mathrm{Na}_{2} \mathrm{O}$ & $\mathrm{REO}$ & $\mathrm{CaF}_{2}$ & Rest & Total \\
\hline 42.6 & 27.2 & 3.0 & 5.5 & 10.6 & 1.43 & 1.22 & 3.61 & 4.07 & 100
\end{tabular}

\section{Results and discussion}

Etching of the glass-ceramics in $2 \mathrm{vol} \%$ hydrofluoric acid (HF) exposed the crystals embedded in glass matrix under the two preparation processes, as shown in Fig. 2 (a) and (b). The micrographs display rod-like polycrystalline granules with similar grain size which are distributed within the glass matrix. The local crystals of the conventional heated sample grow up unusually in three-dimensional, forming cloud-like crystals with diameters of 200-300 nm. According to the energy spectrum analysis of the marked locations in the Fig2 (a) and (b), the mass percentages of the components (as shown in the lower left corner of the figure) are basically similar, but a small amount of $\mathrm{Ce}$ is detected in the crystal phase after microwave treatment. The results of three-point flexural strength test show that the flexural strength for microwave treated sample is $219.9 \mathrm{mpa}$, for conventional treated sample is $169 \mathrm{Mpa}$. The 3D surface morphology of the two materials was measured by laser confocal microscopy, as shown in Fig. 2 (c) and (d). After microwave heat treatment, a high density and uniform bumps are formed in the fracture surface matrix. It indicates that relatively fine and dispersed nanoparticles resist shear force evenly in the amorphous matrix. The fracture surface of conventional treated samples consists of pits and singular bumps with low density. Microwave radiation can promote the uniform growth of crystals due to the special heating pattern and the influence on the diffusion of main nucleating elements. 

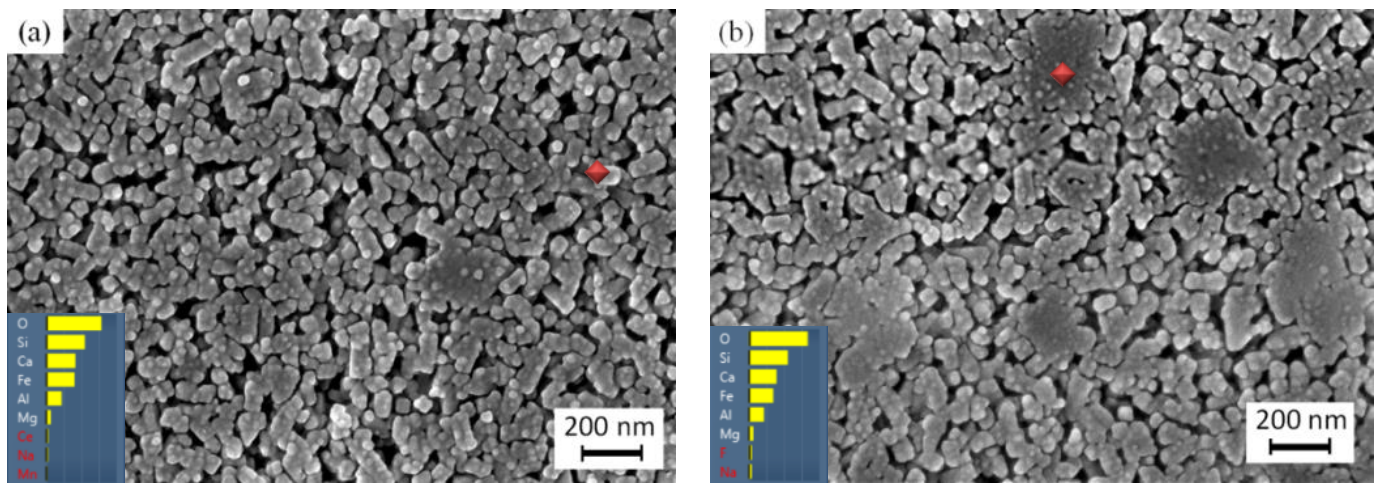

(c)
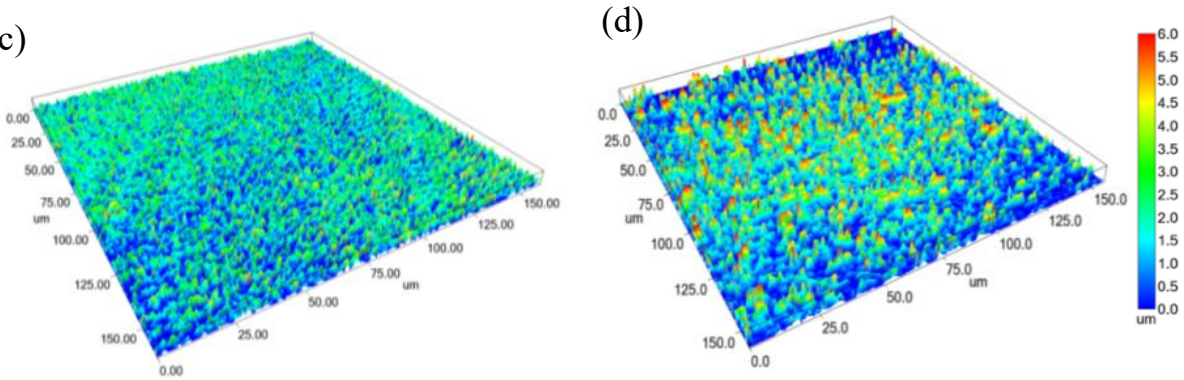

Fig.2 Nanocrystalline glass-ceramics microstructure of conventional and microwave heat treatment

(a)nanocrystalline after microwave heat treatment, (b)nanocrystalline after conventional heat treatment,

(c)the fracture after microwave heat treatment, (d)the fracture after conventional heat treatment.

The research on the fluid-solid interface phenomenon is long-standing, which has been used in engineering application. $\mathrm{ZrO}_{2}$, a hard material with high interface energy, is selected as the substrate[9]. The softening characteristics of materials at high temperature are studied by analyzing the relative projected area $\left(\mathrm{A} / \mathrm{A}_{0}\right)$ and contact angle variation with the temperature[10]. Fig. 3 shows the change of the relative projected area of the samples. The geometrical characteristics are defined as following stages: Local melting $\rightarrow$ Spherical (marked in the figure) $\rightarrow$ Hemispheric $\rightarrow$ Flow, and in turn assigned fixed variation of viscosity. Due to the different thermodynamic parameters between the substrate and the sample, the local melting of the sample results in the increase of the relative projected area with the temperature increasing in the beginning. After that, the initial rectangular projected shape of the precursor is modified to a near-circular shape with enough cohesion for a while before entering the flow state. Each state of microwave heated sample is moved to the high temperature region. Fig. 3(b) shows the curve of contact angle changing with temperature. The contact angles of the two samples varied with temperature in a similar slope, with the maximum values both greater than 100 o, and the wettability seems to have no changed. The hemispherical temperature of microwave and conventional treated samples are $1227{ }_{\circ} \mathrm{C}$ and $1146{ }_{\circ} \mathrm{C}$, respectively. It indicated that the sample prepared by 
microwave has stronger thermal stability at high temperature.

according to the Young's equation[11], which represents the relationship between contact angle and surface tension

$$
\gamma_{l v} \cos \theta=\gamma_{s v}-\gamma_{s l}
$$

Where $\gamma_{s v}, \gamma_{s l}, \gamma_{l v}$ is the solid-vapor, solid-liquid and liquid-vapor interfacial energy, $\theta$ is the measured contact angle. According to this equation, the main driving force of physical wetting by the melt is the surface energy of melt, i.e. when $\gamma_{s v}$ is not high enough, solid phase does not have enough thermodynamic driving force to further reduce its solid-vapor surface energy by spreading melt on it [12]。After microwave treatment, the cohesive force of melt structure is greater, which leads to higher temperature for complete wetting.
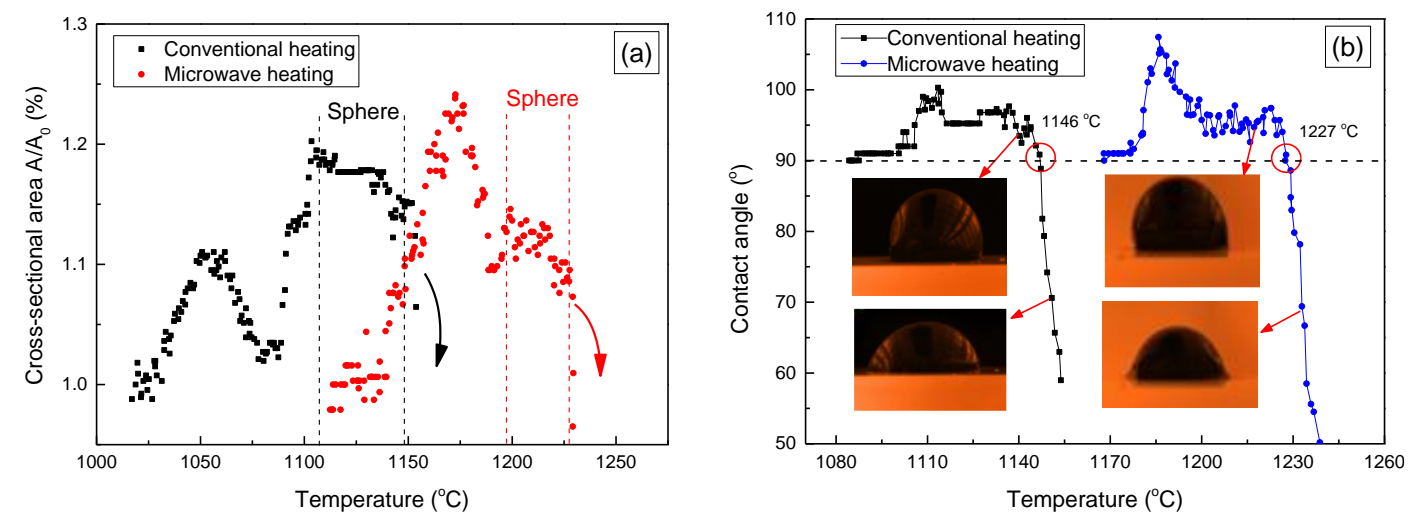

Fig. 3 Melting process of nanocrystalline glass-ceramics

(a) evolution of relative area(A/Ao); (b) evolution of contact angle

Raman bands is caused by the change of molecular polarizability, and its expressive mode is the frequency shift (Stokes or anti-Stokes) related to the initial state of molecules, which can reflect the general characteristics of molecular structure[13]. [ $\left.\mathrm{SiO}_{4}\right]$ tetrahedron is the basic building block of all silicates structure, both of amorphous and crystal. Fig.4 (a) shows the Raman spectra of the as-quenched basic glass precursor material, conventional and microwave treated nanocrystalline glass-ceramics. In the low-wavenumber region, The broad intense band at $\mathrm{P}_{1}$ and $\mathrm{P}_{2}$ is assigned to symmetric Si-O-Si stretching vibrations of the [SiO4] tetrahedron[14] and scissoring bond vibration[15,16].The band position of $\mathrm{P}_{1}$ and $\mathrm{P}_{2}$ has no remarkable changes for the nanocrystalline glass-ceramics samples, while the vibration modes of Si-O-Si band of the tetrahedral network becomes more intense compared with that for the basic glass. The basic glass precursor material retains a strong 
polymeric character. In the process of nucleation heat treatment, the ion migration and phase separation is occurred in the basic glass under the action of high field-strength ions, inevitably disrupting silicon oxygen glass network. The $\mathrm{SiO}_{2}$ content will be reduced due to the precipitation of nanocrystals, which promotes the formation of crystal nuclei. The high-wavenumber region with a wavenumber over 700 cm-1 can reflect the change of the crystal structure. [ $\left.\mathrm{SiO}_{4}\right]$ tetrahedron is a vibrational covalent entity and solid chemical which is easy to establish different tetrahedral arrangements. Q1, $\mathrm{Q}_{2}, \mathrm{Q}_{3}$, and $\mathrm{Q}_{4}$ represent tetrahedra linked with one, two, and three covalent oxygen atoms and completely polymerized silicon-oxygen tetrahedrons (as in pure $\mathrm{SiO}_{2}$ ), respectively. The $\sim 750 \mathrm{~cm}-1$ band is attributed to vibration of isolated tetrahedra $\left(\mathrm{Q}_{0}\right)$ with non-bridge oxygen bond[17]. Fig.3 (b) shows the Si-O stretching $Q_{n}$ components fitted curve for nanocrystalline glass-ceramics in wavenumber of 830-1200 cm-1. A Gaussian shape was assumed for the Raman lines of crystalline moieties, with R-Square above 99.5. The details of main Raman parameters are shown in table 2. Compared with the conventional treated samples, an enhancement of the intensity at $\mathrm{Q}_{2}$ with two bridged oxygen bonds is observed after microwave crystallization, and the intensity of $\mathrm{Q}_{3}$ and $\mathrm{Q}_{4}$ is decreased, pomoting the nucleation as described above. For the wavenumber, Q2, Q3 and Q4 wavenumber of microwave treated samples have a blue-shifting, and the vibration frequency and bond force increase, forming the continuous stable densified nanocrystalline. This provides important evidence for the excellent mechanical properties and thermal stability of the samples after microwave treatment.

Table 2 Band positions of the Raman spectrum

\begin{tabular}{cccccccc}
\hline & $\mathrm{P} 1$ & $\mathrm{P} 2$ & $\mathrm{Q}_{0}$ & $\mathrm{Q}_{1}$ & $\mathrm{Q}_{2}$ & $\mathrm{Q}_{3}$ & $\mathrm{Q}_{4}$ \\
\hline Conventional heating & 521 & 656 & 759 & 873 & 958 & 997 & 1068 \\
Microwave heating & 524 & 657 & 757 & 869 & 962 & 1027 & 1071 \\
Basic glass & 524 & 660 & - & - & - & - & - \\
\hline
\end{tabular}
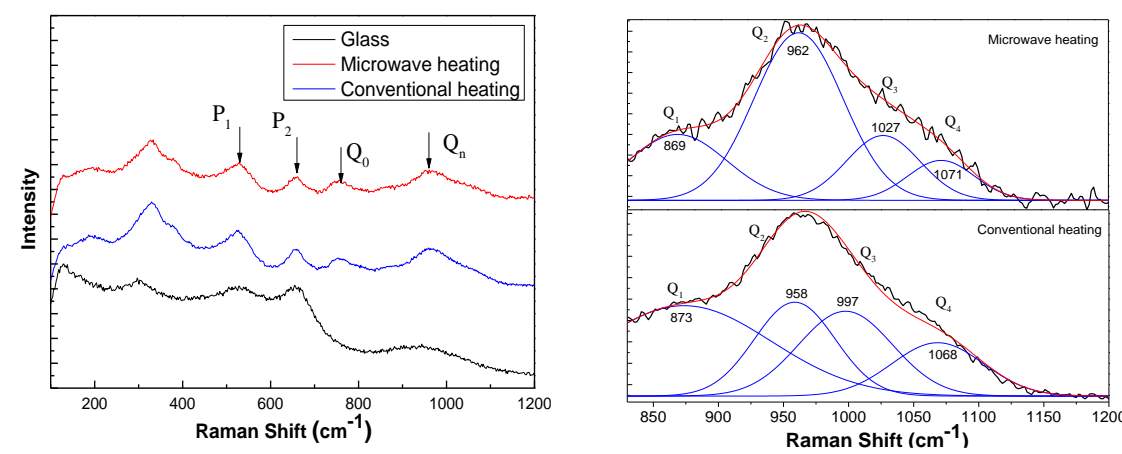

Fig. 4 Molecular structure analysis by Raman spectroscopy 
(a) Raman spectra of nanocrystalline glass-ceramics at room temperature; (b) multi-peak fitting curve in $830 \sim 1200 \mathrm{~cm}-1$ wave number
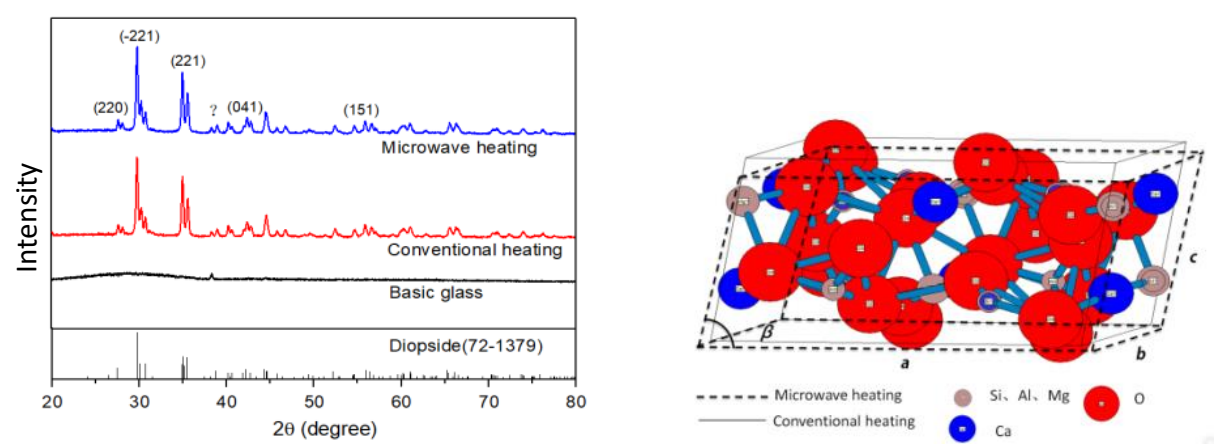

Fig.5 XRD patterns of the basic glass and the samples treated under microwave and conventional heating

(a)XRD pattern; (b) crystal structure schematic

Figure 5 shows XRD patterns of the basic glass and glass samples heat-treated under microwave and conventional heat treatment. The basic glass forms a typical scattering peak at the $2 \theta$ of about 29.40. Nucleation onset temperature is generally above the glass transition temperature $\left(T_{\mathrm{g}}\right)[18]$. After the crystallization heat treatment, diopside crystals are gradually formed in the basic glass[19]. The main characteristics of the amorphous structure are short-range ordered and long-range disordered. The average distance between $\mathrm{n}$ different adjacent molecules or atoms corresponds to the scattering peak intensity and the peak position. According to the quasi Bragg formula of amorphous diffraction[20]:

$$
2 \mathrm{~d} \sin \theta=1.23 \lambda
$$

the average distance between adjacent molecules is about $3.73 \AA$, and the short-range ordered domain of amorphous allowed Schrrer equation:

$$
\mathrm{r}_{\mathrm{s}}=\frac{0.89 \lambda}{B \cos (\theta / 2)}
$$

where, $B$ is the full width at half maxium (rad) of the amorphous scattering peak, which is about $7.979^{\circ}$. The short-range ordered range in basic glass is about $9.85 \AA$, which is also the minimum initial size for forming crystal nuclei. Crystal cell parameters and interplanar spacing were determined by peak position and miller index measured by XRD, as shown in the table 3 . The monoclinic crystal cell of the sample treated with microwave is in the shape of dumpy with a decreased angle of $\beta$. From the chemical bond energy perspective, this crystal structure may be an important factor in enhancing the strengthening of the glass-ceramics. The increased interplanar spacing can be attributed to the 
substitution effectively of different cations during crystallization. The cations with small ionic radii such as $\mathrm{Si}_{4}+(42 \mathrm{pm}), \mathrm{Mg}_{2}+(65 \mathrm{pm})$ and $\mathrm{Al}_{3}+(50 \mathrm{pm})$ in the diopside crystal were replaced by $\mathrm{Ce}_{3+} / \mathrm{Ce}_{4+}(103.4 / 92 \mathrm{pm})[21]$, leading to an increase in interplanar spacing. Several studies have shown that doping Ce ions into crystals can improve thermal stability[22-24].

Table 3 Measured Cell parameters of diopside crystal

\begin{tabular}{llllllllllll}
\hline & & & & & & \multicolumn{4}{c}{ Interplanar spacing $\mathrm{d}(\AA)$} \\
\cline { 7 - 10 } & & $\mathrm{b}$ & $\mathrm{c}$ & $\alpha$ & $\beta$ & $\gamma$ & & $(-221)$ & $(221)$ & $(041)$ \\
\hline JCPDS 72-1379 & 9.794 & 8.906 & 5.319 & 90 & 105.9 & 90 & 3.0017 & 2.5280 & 2.0415 \\
Microwave heating & 9.7848 & 8.8618 & 5.3341 & 90.0 & 106.0 & 90.0 & 2.9976 & 2.5225 & 2.0334 \\
Conventional heating & 9.7798 & 8.8569 & 5.3387 & 90.0 & 106.04 & 90.0 & 2.9976 & 2.5212 & 2.0325 \\
Change rate & 0.05 & 0.06 & -0.09 & 0.00 & -0.04 & 0.00 & 0.00 & 0.05 & 0.04 \\
\hline
\end{tabular}

\section{Conclusions}

The flexural strength and thermal stability of nanocrystalline glass-ceramics with diopside crystal could be improved effectively by microwave heat treatment. From the microstructure evolution, Microwave radiation reduces polymeric character of silicon-oxygen network to promote the nucleation. Q2, Q3 and $\mathrm{Q}_{4}$ wavenumber of microwave treated samples have a blue-shifting, and the vibration frequency and bond force increase, which is also an important reason for the excellent material properties. The structure of diopside cell tended to be dumpy with a decreased $\beta$ angle. Cations with smaller radii are replaced by $\mathrm{Ce}_{3+} / \mathrm{Ce}_{4+}$, leading to increase interplanar spacing, which can improve the thermal stability.

\section{Acknowledgment}

This work is supported by Inner Mongolia University of science and technology innovation fund project under Grant number 2019QDL-B16.

\section{References}

[1] W.H. Sutton, Microwave processing of ceramic materials, Am. Ceram. Soc. Bull. 68 (1989) 376-386.

[2] M. Iwasaki, H. Takizawa, K. Uheda, T. Endo, M. Shimada, Microwave synthesis of LaCrO3, J. 
Mater. Chem. 8 (1998) 2765-2768.

[3] M. Panneer Selvam, K.J. Rao, Microwave synthesis and consolidation of gadolinium aluminum perovskite, a ceramic extraordinaire, Adv. Mater. 12 (2000) 1621-1624.

[4] M.H. Bhat, A. Miura, P. Vinatier, A. Levasseur, K.J. Rao, Microwave synthesis of lithium lanthanum titanate, Solid State Commun. 125 (2003) 557-562.

[5] A. Kaddouri, S. Ifrah, Microwave-assisted synthesis of La1- xBxMnO3. $15(\mathrm{~B}=\mathrm{Sr}, \mathrm{Ag} ; \mathrm{x}=0$ or 0.2 ) via manganese oxides susceptors and their activity in methane combustion, Catal. Commun. 7 (2006) 109-113.

[6] K.Y. Jung, Y.C. Kang, Luminescence comparison of YAG: Ce phosphors prepared by microwave heating and precipitation methods, Phys. B Condens. Matter. 405 (2010) 16151618.

[7] J. Binner, B. Vaidhyanathan, A. Paul, K. Annaporani, B. Raghupathy, Compositional effects in nanostructured yttria partially stabilized zirconia, Int. J. Appl. Ceram. Technol. 8 (2011) 766782.

[8] L. Baowei, D. Yongsheng, Z. Xuefeng, J. Xiaolin, Z. Ming, C. Hua, Effects of Iron Oxide on the Crystallization Kinetics of Baiyunebo Tailing Glass-Ceramics, Trans. Indian Ceram. Soc. 72 (2013) 119-123.

[9] Y. KAWAI, M. UO, F. WATARI, Microstructure evaluation of the interface between dental zirconia ceramics and veneering porcelain, Nano Biomed. 2 (2010) 31-36.

[10] I. Duarte, J. Ferreira, Evolution of metallic foams using hot-stage microscopy, Procedia Mater. Sci. 4 (2014) 251-256.

[11] H. Tavana, A.W. Neumann, Recent progress in the determination of solid surface tensions from contact angles, Adv. Colloid Interface Sci. 132 (2007) 1-32.

[12] M.T. Marefati, A.M. Hadian, T. Hooshmand, A. Hadian, B.E. Yekta, Wetting characteristics of a nano Y-TZP dental ceramic by a molten feldspathic veneer, Procedia Mater. Sci. 11 (2015) $157-161$.

[13] A. Chakrabarti, A.R. Molla, Eu3+ doped ferroelectric CaBi2Ta2O9 based glass-ceramic nanocomposites: Crystallization kinetics, optical and dielectric properties, Ceram. Int. 44 (2018) $7557-7568$.

[14] S.J.L. Ribeiro, P. Dugat, D. Avignant, J. Dexpert-Ghys, Structure and crystallization of 
lanthanum fluorozirconate glasses, J. Non. Cryst. Solids. 197 (1996) 8-18.

[15] K.E. Lipinska-Kalita, S.A. Gramsch, P.E. Kalita, R.J. Hemley, In situ Raman scattering studies of high-pressure stability and transformations in the matrix of a nanostructured glass-ceramic composite, J. Raman Spectrosc. 36 (2005) 938-945.

[16] B. Li, S. OUYANG, X. ZHANG, X. Jia, L. Deng, F. Liu, Effect of temperature on the structure of $\mathrm{CaO}-\mathrm{MgO}-\mathrm{A} 12 \mathrm{O} 3-\mathrm{SiO} 2$ nanocrystalline glass-ceramics studied by Raman spectroscopy, Spectrosc. Spectr. Anal. 34 (2014) 1869-1872.

[17] P. Colomban, A. Tournie, L. Bellot-Gurlet, Raman identification of glassy silicates used in ceramics, glass and jewellery: a tentative differentiation guide, J. Raman Spectrosc. 37 (2006) 841-852.

[18] H. Xiao, Y. Cheng, L. Yu, H. Liu, A study on the preparation of CMAS glass-ceramics by in situ crystallization, Mater. Sci. Eng. A. 431 (2006) 191-195.

[19] S.S. Jiang, L. Zhu, H. Zheng, Y.G. Wang, Kinetics of non-isothermal crystallization in FeNiPC (Nb) Alloys, Thermochim. Acta. 684 (2019) 178481.

[20] J. Yiqin, J. Yugang, L. Huasong, W. Lishuan, L. Dandan, J. Chenghui, Y. Yaping, F. Rongwei, C. Deying, Analysis on effects of thermal treatment on structural characteristic of ion beam sputtering SiO2 films, Infrared Laser Eng. 42 (2013) 418-422.

[21] R.D. Shannon, Revised effective ionic radii and systematic studies of interatomic distances in halides and chalcogenides, Acta Crystallogr. Sect. A Cryst. Physics, Diffraction, Theor. Gen. Crystallogr. 32 (1976) 751-767.

[22] C.M. Murphy, H. Ravner, The effect of cerium on the thermal stability of polysiloxanes, J. Polym. Sci. Part B Polym. Lett. 2 (1964) 715-718.

[23] J. Di, Y. Jing, C. Yi, D. Su, Effects of Doping Yttrium and Cerium on Thermal Stability of Sepiolite, J. Chinese Rare Earth Soc. 20 (2002) 112-115.

[24] M. Ozawa, M. Kimura, A. Isogai, Thermal stability and characterization of $\gamma$-Al2O3 modified with rare earths, J. Less Common Met. 162 (n.d.) 297-308. 